\title{
Retinal Detachments-When to Refer? Identifying the Complicated Case
}

\author{
P. K. LEAVER \\ London
}

\begin{abstract}
Summary
Cases of retinal detachment are usually referred by one ophthalmologist to another, because of their complexity. The complicated nature of such cases can be judged by their likely response to conventional methods of retinal reattachment surgery. When the causative retinal breaks cannot be identified or closed by conventional means, it is reasonable to call detachments complicated. The prompt identification of such cases and their referral, if appropriate facilities and expertise to undertake them are not at hand, are essential steps in the correct management of rhegmatogenous retinal detachments.
\end{abstract}

The decision to refer a patient with retinal detachment is usually taken because the referring ophthalmologist considers the case is (or has become) more complicated than he or she feels confident to treat. Management of "simple" retinal detachments is undertaken with confidence by the majority of ophthalmologists in the United Kingdom, "simple" being the term commonly applied to retinal detachments which can be expected to respond to conventional methods of repair with a high degree of certainty.

Considering then, that the pathogenesis of retinal detachments is well understood, why do they not all respond favourably to surgery at the first attempt? The answer is twofold: (i) Because the surgical method was incorrectly applied or (ii) Because it was wrongly chosen. In the first instance it may be possible to correct the mistake at a second operation without prejudice to a successful outcome, but careful judgement is needed to be sure that a further conventional procedure is appropriate. In the second, it is essential to recognise that the diagnosis was wrong and that therefore the detachment will not respond to further conventional techniques. In either case the identification of signs leading to a diagnosis of complicated retinal detachment is of crucial importance, since ill-judged attempts at retinal reattachment by inappropriate means inevitably lead to further complications.

A retinal detachment is defined here as complicated if the break(s) cannot be found and closed by conventional methods of reattachment surgery (Figure). It is the purpose of this paper to help identify those factors which determine whether or not conventional retinal reattachment surgery (using scleral buckling and cryotherapy with or without drainage of subretinal fluid) is likely to be effective. To do this we must first be able to answer two further questions: (1) Can (all) the break(s) be identified? (2) Is there a reasonable prospect of closing them using conventional methods? The answers to these questions are by no means mutually exclusive, for in some instances, although the breaks can be easily 




Fig. 1. Flow-chart illustrating pathways along which the diagnosis and management of retinal detachments (RDs) should proceed.

identified, there is little chance of closing them by conventional means, while in others, it is possible to close unseen breaks conventionally.

\section{Identification of breaks}

Failure to identify (and therefore treat) all of the breaks usually results in surgical failure, while unsuccessful attempts at surgery encourage the development of proliferative vitreoretinopathy (PVR), so that accurate identification of breaks has a major bearing on the ultimate prognosis.

There are three reasons for difficulty in finding retinal breaks:

(1) Their small size, eg very small breaks in aphakic eyes.

(2) Their location, especially where the breaks are very anterior or are buried within retinal folds.

(3) Opacities in the ocular media, usually in the lens or vitreous.
To find and seal all of the breaks is the aim of every retinal reattachment operation. Their identification is therefore of the utmost importance. A retinal detachment in which the break(s) are not readily observable is difficult to treat successfully by conventional means and must be viewed as a complicated case until proved otherwise.

A retinal detachment which has failed to respond to conventional surgery must similarly be regarded as complicated unless it is plain that the operative technique was at fault and can reasonably be remedied by another conventional procedure. Persistence or recurrence of retinal detachment is always due to the presence of an open break, whether it be the original one(s) or another. Any one or a combination of several factors may be responsible for the presence of the open break(s) and the cause must be identified before the direction of further management can be decided. 


\section{Failure to find a break}

(i) In eyes with clear media

While retinal detachments in otherwise healthy patients are occasionally non-rhegmatogenous, the presence of "tobacco-dust" in the vitreous and the characteristics of the detachment itself are usually diagnostic, so that failure to identify a break is no reason to assume that one is not present.

When the fundus can be seen without difficulty it can usually be assumed that the break(s) are too small to see or are hidden by folds in the detached retina. A macular hole is particularly likely to be missed because of the latter and can sometimes only be seen by examining the patient in different positions, for instance lying flat or sitting up. In most cases, however, posterior breaks are easily seen and if none is identified it can be assumed that the culprit(s) are small and very anteriorly situated. This is particularly common in aphakic eyes.

Sometimes the position of the unseen break can be predicted with confidence from the history, topography and other characteristics of the detachment, using Lincoff's "rules"1 and a conventional buckling procedure can be used with reasonable optimism and safety. Detachments with very small breaks are in any case less likely to develop PVR than those with large ones, so this course of action may be reasonable in the first instance, especially if it is felt that a more aggressive approach is unlikely to make the break(s) easier to find.

In other cases, however, it may be wiser to opt for a closed microsurgical method in the first instance to facilitate an internal search. ${ }^{2}$ The advent of closed intraocular microsurgery and its relative safety in experienced hands has made a helpful contribution to the management of complicated detachments and it is becoming increasingly difficult to justify a "hit and miss" approach when the breaks cannot be identified prior to surgery.

\section{(ii) In eyes with opacities in the media}

Opacities in the ocular media of eyes with retinal detachments are not uncommon. Vitreous haemorrhage, as a direct consequence of a retinal tear, may be profuse and obscure all details of the fundus, while pos- terior vitreous detachment in cases of branch retinal vein occlusion, proliferative diabetic retinopathy and sickle-cell disease may simultaneously precipitate retinal detachment and dense vitreous haemorrhage. Early diagnosis of retinal detachment in such cases is essential and is based on the history (of increasing visual deterioration), the development of an afferent pupillary defect and results of ultrasound investigation. Rapid surgical intervention, using closed intraocular microsurgical methods, has improved the prognosis, because it is possible, by reattaching the retina quickly, to preempt the development of PVR, in eyes with a combination of intravitreal haemorrhage, retinal tears and detachment.

Intravitreal cells and debris obscuring details of retinal detachment occur, though less commonly, in other circumstances, notably in association with long-standing retinal detachments, acute retinal necrosis and chorioretinitis from other causes leading to secondary rhegmatogenous retinal detachment. Not only may such inflammatory states give rise to intravitreal debris, but deposits on the lens capsule, together with posterior synechiae, may make conventional retinal reattachment surgery difficult, if not impossible.

Small peripheral retinal breaks in the eye of an elderly patient with lens opacities can be hard to find. Removal of the lens at the time of retinal surgery complicates the operation and may render little or no improvement in the view, especially if the pupil constricts after cataract extraction. In such a case, a conventional approach utilising the Lincoff principles is often the method of first choice, offering as it does a non-invasive, safe procedure which is unlikely to jeopardise the chances of subsequent surgery, if this proves to be necessary. Similarly, in pseudophakic eyes, where clear visualisation of the peripheral fundus is often difficult and vitrectomy with internal tamponade complicated by the presence of the lens implant, an initial attempt at retinal reattachment using conventional methods (aided by Lincoff's principles regarding the probable location of the breaks), is often justified. Should this operation fail, however, a closed microsurgical approach is indicated. Removal of the intra- 
ocular lens implant, in such a case, is rarely necessary or advisable.

Occasionally, corneal opacities obscure the fundus view in cases of retinal detachment. The application of conventional methods, as already described, is always justified if the topography of the detachment can be identified, because the difficulties of undertaking corneal transplantation combined with retinal reattachment surgery, are considerable.

\section{Closure of breaks}

Retinal detachments with the following characteristics, either alone or in combination, commonly fail to respond to conventional methods of reattachment surgery:

(1) The breaks are difficult to identify (Discussed above).

(2) The breaks are very large.

(3) The breaks are very posterior, eg macular holes.

(4) The breaks are numerous and in differing meridia.

(5) A giant retinal tear is present.

(6) The vitreous gel has collapsed, eg in high myopes.

(7) There is traction on the break(s) due to incarcerated vitreous, local epiretinal membranes or PVR.

It is generally understood that scleral buckling beneath retinal breaks encourages retinal reattachment by relieving vitreous traction on the break and by a prejudicial effect on transport of fluid through the break in favour of the pigment epithelial pump, induced by a plugging effect of the vitreous gel. ${ }^{3}$ Conventional buckling techniques commonly fail when used to treat retinal detachments with the characteristics listed above, for the reasons discussed below.

\section{Large breaks}

Very large tears are not easily plugged by the gel because the vitreous is frequently collapsed and the detached retina in consequence very bullous. Drainage of subretinal fluid (SRF) is almost always necessary and scleral buckling without internal tamponade leads inevitably to fishmouthing of the posterior flap and the formation of retinal folds. Failure to close such large breaks at the first attempt carries with it a very high risk of inducing PVR. These cases are therefore best regarded as complicated at the outset and treated accordingly.

\section{Posterior breaks}

Posterior breaks are difficult to manage by conventional methods, because of difficulties associated with gaining access to the posterior sclera and achieving an adequate buckle. They occur commonly in highly myopic eyes and, like giant tears, are frequently associated with disorders of the vitreous collagen and vitreoretinal interface, such as Stickler's Syndrome. In consequence, they are ideally suited to an internal approach and are usually best managed in this way.

\section{Multiple breaks}

Detachments associated with multiple retinal breaks are not always difficult to treat by conventional methods and are not therefore necessarily complicated. In many instances, however, the number, size and distribution of the breaks is such that they are difficult to close without very extensive buckles combined with drainage of SRF. This type of surgery carries a high risk of complications, while the outcome in terms of retinal reattachment is by no means certain, so it is important to identify those cases in which conventional techniques are likely to fail and treat them by an internal approach.

\section{Giant tears}

Giant retinal tears are difficult to close by conventional means, because of the size and independent mobility of the posterior flap, and the presence of vitreous behind it. The risk of PVR is high, even in the event of successful reattachment, while the consequences of failed surgery are disastrous. These cases should always be recognised as complicated at the outset and treated by closed microsurgical techniques.

\section{Vitreous collapse}

The extent and depth of retinal detachment do not in themselves make a case complicated. Collapse of the vitreous gel, however, leading to hugely bullous detachment with deep retinal folds, sometimes obscuring the break(s), carries a doubtful prognosis for suc- 
cessful reattachment by conventional buckling methods. Internal tamponade is usually necessary and closed intraocular microsurgery is frequently essential in such cases (often highly myopic eyes), especially following cataract extraction. In such cases, when there is difficulty in identifying the break(s) and/or being able to close them with certainty, the use of an internal approach should be considered as a primary procedure.

\section{Traction}

The identification of tractional forces contributing to the occurrence, persistence or recurrence of retinal detachment following conventional surgery, is becoming increasingly common. Occasionally, in eyes which have undergone previous surgery or penetrating injury, vitreous incarceration into the section, SRF drainage-site or scleral wound, causing direct traction on the retinal break(s), is obvious and the indication for intraocular microsurgery is clear. More commonly, however, the development of tractional forces is subtle and insidious, evolving as it does from the gradual proliferation and contraction of epiretinal membranes, either locally or widely throughout the fundus.

The identification of these signs and the resultant change in diagnosis from "simple" to complicated retinal detachment, at an early stage in their development, is crucial to the management and subsequent outcome of the case. It should be emphasised, however, that the mere presence of retinal folds with limited mobility and/or membranes, which do not prevent closure of the break(s) by scleral buckling, with or without drainage of SRF, is not necessarily an indication for complicated intraocular manoeuvres.

\section{Conclusions}

By defining a complicated case as one which cannot reasonably be expected to respond to conventional methods of retinal reattachment (Figure), the question: "when to refer?" can more easily be answered. If identification of retinal breaks and/or their successful closure by methods locally available, is in doubt, the case is complicated and should be referred to a centre where there are appropriate facilities. As our knowledge, understanding and technical skills increase, the definition of "simple" and "complicated" retinal detachments will change and so will the indications to refer.

I am grateful to the Department of Medical Illustration, Moorfields Eye Hospital, for their help with the preparation of the Figure.

\section{References}

${ }^{1}$ Lincoff $\mathrm{H}$ and Gieser R: Finding the retinal hole. Arch Ophthalmol 1971, 85: 565-9.

${ }^{2}$ Rosen PH, Wong HC, McLeod D: Indentation microsurgery: internal searching for retinal breaks. Eye 1989 (in press).

${ }^{3}$ Foulds WS: The vitreous in retinal detachment. Trans Ophthalmol Soc UK 1975, 95: 412-6. 